\title{
The Technology System Framework of the Internet of Things and Its Application Research in Agriculture
}

\author{
Hong Zhou ${ }^{*}$, BingWu Liu, and PingPing Dong \\ School of Information Science \& Technology, Beijing Wuzi University, Beijing, China
}

\begin{abstract}
Directing at the current development condition of the internet of things and based on the available technology analysis of the internet of things, the paper makes analysis and research on the internet of things in terms of technological levels and systems. Started from three aspects, respectively, data collection, network service, date fusion and computation, the paper analyzed the technologies like RFID, ZigBee, sensors, Cloud Computing and so on, based on which the paper further brought forth the technological system framework of the internet of things. By combining the technology system framework, the paper carried out the application research in aspect of intelligent agriculture and raised the system construction of production monitoring system of agriculture standardization which is based on the internet of things. Moreover, the paper carried analysis and research works on the sensor nodes of the system, made analysis and discussion on the various technologies involved.
\end{abstract}

Keywords: the internet of things, intelligent agriculture, information collection, ZigBee, Sensor.

\section{Introduction}

Internet of Things refers to a network allows a series of intelligent activities like identification, positioning, tracking, monitoring and management by linking devices like RFID, Smart Sense, GPS (Global Positioning System) and 2-D Code, etc. in objects to wireless network via interfaces to endow objects with intelligence, therefore realize the communication and dialogue between human and objects as well as objects and objects.

IOT and relevant technologies have already been highly values by enterprises and in academic circles both inside and outside the country. Moreover, series of research and exploring works have been launched. However, both inside and outside the country, IOT's research and development works still remain in a preliminary condition and their architecture and system model haven't been developed. There are certain blindness in the research and development of the IOT technology. In terms of the proper definition, the fundamental principles, the architecture and the system

\footnotetext{
"Supported by Funding Project for Academic Human Resources Development in Institutions of Higher Learning Under the Jurisdiction of Beijing Municipality (PHR200906210), Beijing Social Science Project(09BAJG258), Beijing-funded project to develop talents (PYZZ090420001451).
} 
model of IOT, there are plenty of questions to be considered and discussed. Based on the current IOT technology analysis, by analyzing and discussing the technological levels and systems of IOT, the paper is going to start the research on the architecture and the framework of IOT.

Started from the intelligent transportation, logistic scheduling and tracing and base station monitoring, IOT extends its application domain to public oriented personal medical treatment, intelligent home furnishing and so on, and its applications can be found in all walks of life. However, being in the preliminary stage and asking for innovations, IOT hasn't been popularized in large scales. The IOT industry covers sensors, transmission tunnels, computation and process, industrial application and so on, which involves technologies like RFID, sensors, wireless network transmission, computation with high-performance, intelligent control and so on. The paper is going to unfold the introduction by combing the application of IOT in the monitoring system for the agricultural standardized production.

\section{Key Technologies of IOT}

Internet of Things is an open architecture with a variety of technical supports, including radio frequency identification technology, middleware technology, logistics management, e-commerce technology and so on. It involves three key technologies: (1) sensing technology--obtain the object information simultaneously and accurately through the use of RFID, sensor, two-dimensional product code and other equipments and technologies; (2) information transfer technology-- deliver real-time and accurate object information by using the deep integration between a variety of telecommunications networks and the Internet; (3) intelligent processing technology-analyze and sort out large amounts of information and data as well as implement intelligent control of the goods through cloud computing and fuzzy intelligent recognition technology. Considering the content of IOT, in the following part, we are mainly focused on analyzing and researching the information collection technology (ID identification, location information), network service technology (wireless sensor networks, core network element and so on), data merger and computation technology (Cloud computing, mass data merger) and so on.

\subsection{Technology of Information Collection}

The gathering technology of IOT is sensing technology which is the foundation of IOT. Currently, information collection mainly depends on electronic tags and sensors, etc. In sensing technology, the electronic tag is used to standardize the identification of the collected information; and the data acquisition and device control are realized through radio frequency identification and two-dimensional code readers, etc. In data collection and processing stages, it mainly uses various types of sensor technologies, RFID, two-dimensional code and other information gathering techniques to collect data and then receive the control signals from upper receiver, respond, and complete the corresponding actions to process information.

RFID is a non-contact automatic identification technology and it identifies targets automatically and collects relevant data by radio-frequency signal. By affixing 
electronic labels to the objects, RFID realizes high efficient and flexible management and it becomes the most critical technology for IOT. Typical RFID system is comprised by electronic labels, readers and information process system. Without the manual involvement, the identification process is capable of all kinds of severe environment. RFID is able to identify high-speed traveling object and multiple labels at the same time, with great convenience and efficiency in operation. By absorbing technologies like internet, communication and so on, RFID realizes the object tracing and the information sharing all around the world.

Currently, in the field of RFID, most of researches are based on label cost, uniform technology standard, key technology and so on. Besides RFID, the sensor technology is another frequently applied technology in the ITO. Sensor refers to devices and equipment which are able to perceive the determined test object and the transform it into useful signals in accordance with certain rules. In most cases, the sensor is comprised by sensitive element and conversion element, which are able to detect, perceive external signals, physical and chemical and physical conditions. Meanwhile, the sensor technology is the premise of the sensor node technology.

\subsection{Technology of Network Communication}

In communication technologies of IOT, there are a variety of technologies to choose. They are mainly divided into two types, wired technology (e.g. DSL, PON, etc.) and wireless technology (e.g. CDMA, GPRS, IEEE 802.11a/b/g and WLAN, etc.), which are relatively mature. Referring to the implementation of IOT, the wireless sensor network technology is particularly important.

WSN is a network system which integrates distributed data collection, transmission and process technology and it is widely concerned for its network method and installation method with low cost microminiaturization, feasibility, reliability and flexibility and its capacity for moving object. By sensor node and networks spread in different place, IOT is able to perceive the world. The network construction of WSN can be classified into physical layer, data link layer, internet layer, transmission layer and application layer. The basic composition of sensor's network node includes sense unit, process unit, communication unit and energy unit.

In the technologies of IOT's network and communication, ZigBee is not only a technology between wireless labeling technology and Bluetooth, but also a bidirectional wireless communication technology with close range, low complexity, low power consumption, low cost. ZigBee is applicable in the domain of automatic control and long-range control. ZigBee, as a transmission standard, can be applied in multiple frequencies and working segments and is widely applied for its low power consumption and low cost. In domains of safety system, sensor network, industrial monitoring and other IOT areas, ZigBee has great development space. Moreover, by integrating IPv6 technology into the IOT, we can realize the end to end communication with the current network equipment and improve the retransmission efficiency, which further enhance the safety of information transmission.

\subsection{Technology of Data Fusion and Computing}

IOT is comprised by numerous nodes in the sensor network. In the process of information perception, it is not feasible to adopt the method of single nodes 
transmitting the date to the sink independently. Because there is enormous redundant information, they would waste much communication width and precious energy resource. Besides, they would lower the efficiency of information collection and affect the timeliness of information collection. For this reason, we need to adopt data merger and intelligent technology to deal with the problem, during which distributed data merger and cloud computing are involved.

Cloud computing is one of distributed computing. By automatically dissembling the huge computing and processing processors into countless subprograms, it handled these subprograms to the huge system composed by multiple servers for searching, computing and analysis, after which it transfers the procession result to the user. Via this technology, web service providers is able to process millions and millions, even billions and millions of information, and thus be capable of net service as powerful as "super computer". By taking advantage of the low cost, super powerful procession ability and storage ability of Cloud Computing Center, and the everywhere information collection of IOT, we combined them together, and enable all kinds of objects to exchange and communicate, thus realizing the intelligent identification. By means of visualization technologies, single server can support multiple virtual machines in running multiple operating systems, and therefore improving the use ratio of servers. Cloud Computing is a service which provides those dynamic, scalable and visualized computing resources by internet.

Visualization, elastic range extension, distributed storage, distributed computing and multiple lessees are critical technologies of Cloud Computing. Distributed storage aims at the target of satisfying the storage requirement which can't be satisfied by single server by utilizing the storage resources of multiple servers inside the cloud environment, and its characteristic is that storage resource is able to be expressed abstractly and be managed in uniform. Moreover, it is capable of ensuring the security and reliability and other requirements generated in the data read-write and operation.

\section{Processor Architecture of IOT System}

Based on the research and analysis on IOT's critical technologies, in the general technological system architecture of IOT, we shall ensure the size of IOT (only with certain size can we enable the object intelligence to play their role), mobility (we need to ensure the object can realize instant communication under moving condition even with high speed ) and security (as for those which involve national security, trade secret and personal privacy, core technology with proprietary intellectual property rights are necessary) The application of IOT is still relatively fragmented in China, and there is no large-scale, systematic development trend, so a scalable and open architecture of IOT should be established in the industrial chain technology as well as application and formation system in ideal aspects in order to break through the barriers of application in large-scale and promote the IOT industry's transition from the start-up period to the growth period. The overall technical architecture of IOT includes data acquisition layer, information exchange layer and application layer. The overall technical architecture of IOT is shown in Figure 1. 
Data collection layer consists of two-dimensional code tags and readers, RFID tags and readers, cameras, sensors, GPS, sensor gateways, sensor networks and other equipment and technologies. In this layer, it mainly solves the issues like data collection and object identification, etc. It is composed by the various types of acquisition and control modules, and the main function is to complete IOT's information perception, data collection and the control of facilities. It is an important foundation of IOT.

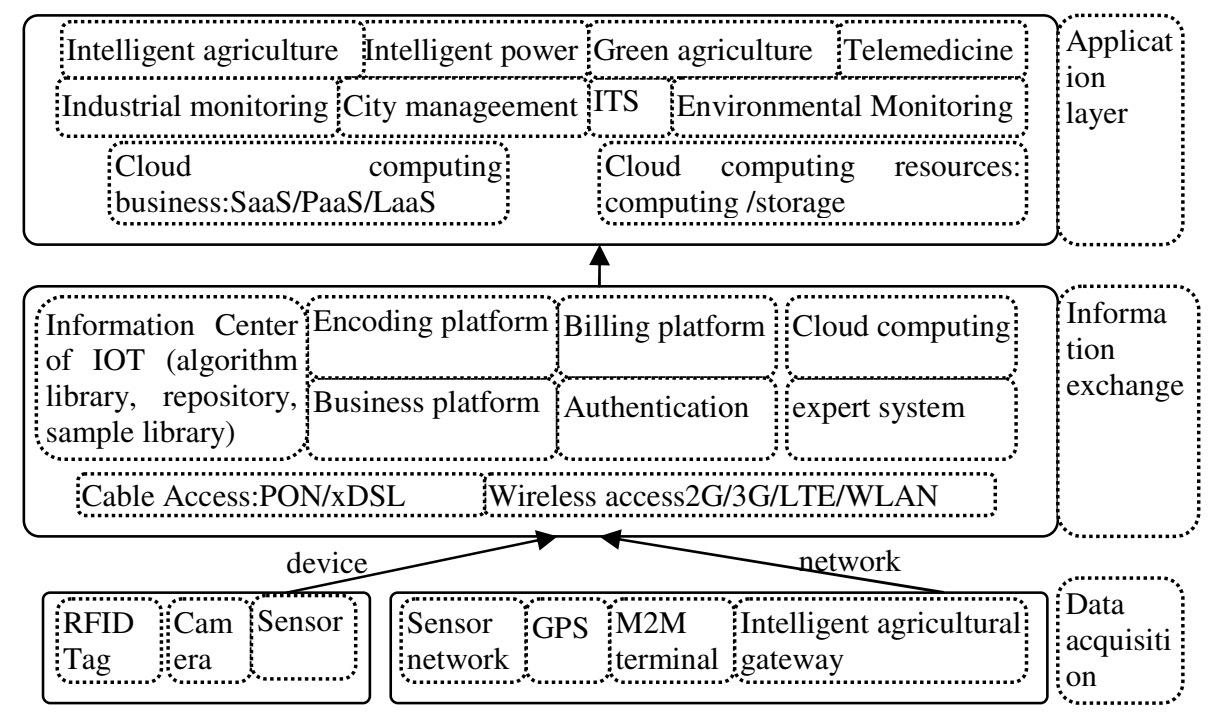

Fig. 1. The technical architecture of IOT

The layer of information exchange is based on the network of IOT and communication technologies, such as mobile communication network and the Internet, which is a converged network formed by a variety of communication networks and the Internet. It includes information center, management center of IOT, expert systems and cloud computing platform, which are responsible for the massive part of Intelligent Information Processing. Therefore, the network layer not only requires the ability to operate the network, but also the ability to enhance operational efficiency of information. It is the infrastructure to make the IOT become a universal service.

Application layer refers to solutions of integrating IOT technologies with industrial technical expertise to achieve a wide range of application with intelligent technologies. Through the application layer, IOT ultimately realizes deep integration with information technology and industrial professional technologies. The application layer lies on the top which is the ultimate goal of IOT applications. It consists of a variety of servers and its main functions include the collection, transformation and analysis of the gathered data as well as the adaptation and triggers of things for users. The key issue in this layer is socialization of information's sharing and information security. 


\section{Monitoring System for Agricultural Standardized Production Based on IOT}

In the process of agricultural production, the most critical part is the true time data collection in terms of temperature, moisture, carbon dioxide content, and soil temperature and soil moisture content. By making use of the IOT platform and GPRS/TD, by means of SMS, WEB, WAP and other methods, we can make the users dealing with agricultural production acquire these real-time information. Monitoring System for Agricultural Standardized Production based on IOT aims at the target of making information collection towards several indexes in crop growing and carrying out systemic monitoring towards the plantation area, crop pattern, crop growing, the breaking out and development of agricultural damages, crop output and so on.

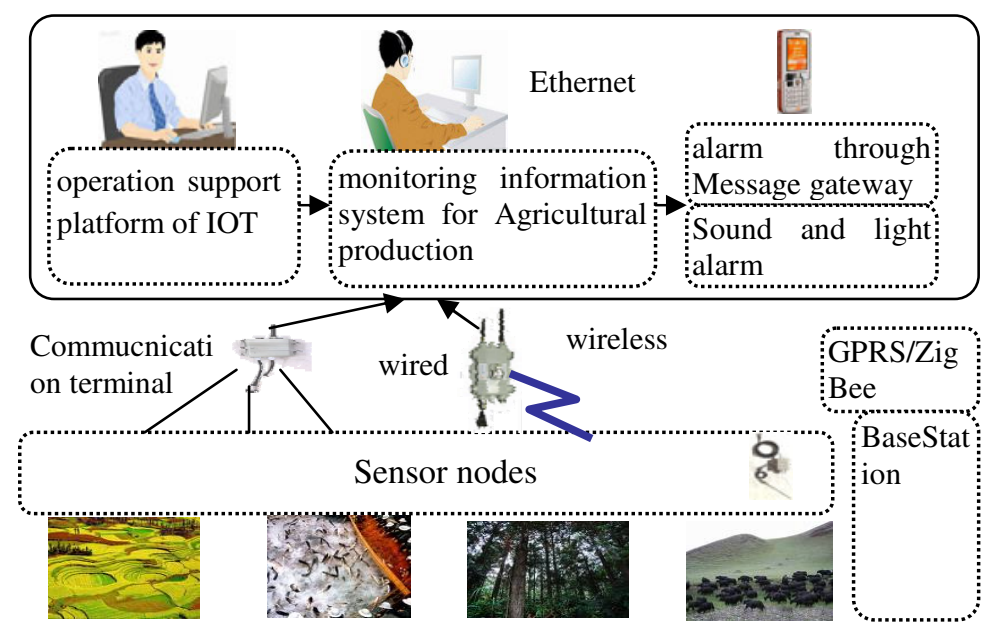

Fig. 2. The system architecture of Monitoring system

The monitoring system is composed by the wireless sensor monitoring network and distant monitoring information system. ZigBee sensor nodes set in the plastics tents or greenhouse collect the critical index in crop growing like air temperature, moisture, soil temperature, moisture, illumination intensity, $\mathrm{CO} 2$ concentration and so on. Moreover, by means of ZigBee short range wireless communication technologies, we can realize the date transmission and transmit all the node date to the base state where we can carry out TCP/IP packaging on the data we've received, after which we can send them to the remote control center by means of GORS. The control center receives data, analyze and express them. Moreover, they can make parameter setting. Once environmental parameters go beyond the set values, it is possible to make acousto-optic alarming or message alarming. By making use of cell phone or remote computer, researchers can make real-time control on the environmental conditions and information in the crop growing spot. The architecture of monitoring system is shown by the Picture 2 . 
Monitoring system is composed by three parts: (1) sensor node, sending the information like atmosphere collected by the senor in periodicity to the monitoring and management center of agricultural environment by means of multiple hop transmission. (2)gate way. Located in the edge of sensor network. Realizing the interconnection and communication between the sensor network and internet. In the gateway, we can realize the conversion from the sensor network protocol to the internet protocol. (3) Monitoring and management center of agriculture environment (user), being responsible for the information storage, procession, evaluation and so on. One management center usually is capable of managing multiple monitoring areas. Remote control and PDA users are able to visit the data of the environmental monitoring center by means of internet and they can make real-time inquiry via the center.

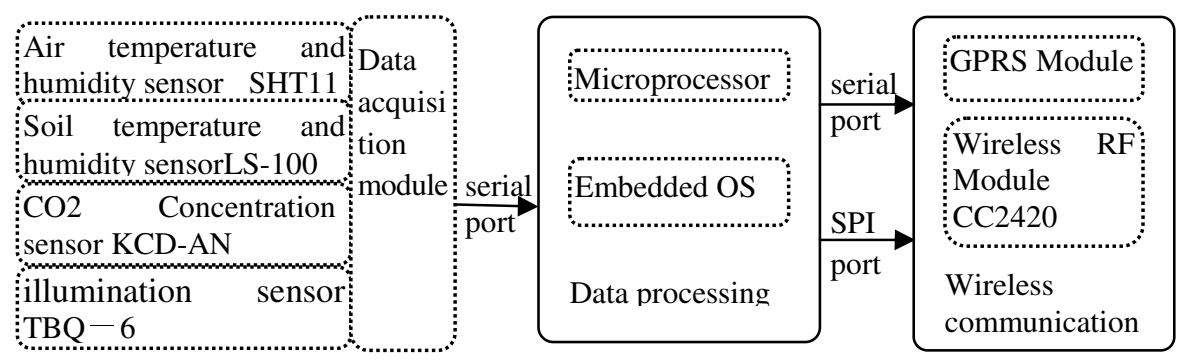

Fig. 3. Structural framework figure of the sensor nodes

Sensor network is basically comprised by sensor board which is set with sensors of air temperature and moisture, soil moisture and temperature, soil $\mathrm{PH}$ value, light intensity and $\mathrm{CO} 2$ concentration. These sensors can be selected and set in the sensor board. The sensor board makes adjustment on the input signals of sensors which is collected by the terminal node of wireless sensor network. The general architecture of sensor node is shown by picture 3. Temperature and moisture sensors are more and more widely applied in the areas of industrial and agricultural production, whether, environment protection and so on. We've selected the SHT11, a digital moisture and temperature sensor with high-accuracy, which is produced by Sensirion in Switzerland. The sensor is a new typed and digital relative moisture and temperature sensor with two passes of serial interface. The sensor has monolithic calibration and can be used to measure relative moisture, temperature, dew point and other parameter, with characteristics of digital output, adjustment free, standardization free, peripheral circuit free, good exchanges. As for the air moisture and temperature sensor, we've adopted SHT11, digital moisture and temperature sensor with high accuracy, which is produced by SENSIRION in Switzerland. As for the soil temperature moisture sensor, we've adopted the LS-100, soil moisture sensor, which is produced by domestic companies. As for the $\mathrm{CO} 2$ concentration sensor, we've adopted KCD-AN, produced by Korean companies.

Data procession module is comprised by micro processor, data storage circuit and embedded operation system and it is the core component of sensor node. Moreover, it is responsible for data's storage and procession, scheduling system tasks, carrying out the communication protocols and so on. Wireless communication module is responsible for the communication among the sensor nodes and between the nodes 
and the station, information exchange and control among sensor nodes and the data collected by the receiving and sending station. When design the system, we adopted CC2420 ship which supports ZigBee protocols, with a frequency band of $2.4 \mathrm{GH} \mathrm{z}$. we've adopted DSSS (Direct Sequence Spread Spectrum) under the specification of IEEE 802.25.4.

\section{Conclusion}

The paper has analyzed the current situation of IOT and made analytical research on the major technologies of IOT. Directing at the practical requirement of national agricultural environment, we've design a agricultural standardized production monitoring system based on the IOT. The system can realize the automatic configuration and self organized transmission of information collected node, realized the real-time collection, transmission, expression and storage of agricultural environmental information. By applying it to the construction agriculture, we can better solve the defect in the traditional monitoring system of green house and plastic tents and make people acquire agricultural environment information at any time. Moreover, we can realize the remote, real-time and accurate monitoring on the agricultural environment. In many aspects, IOT has displayed its promising research and application values. What is more, its application in agriculture has become one of hotspots because it constructed a precious technological platform for the precision agriculture in turning from demonstration to the practices. IOT enables traditional agricultural pattern to evolve into a information network centered production mode and brings automation, networking and intelligentializing to the agricultural production.

\section{References}

1. Cai, B., Bi, Q.-S., Li, F.-C., Wang, D., Yang, Y., Yuan, C.: Research and Design of Agricultural Environment Monitoring System Based on ZigBee Wireless Sensor Network. Acta Agriculturae Jiangxi (11) (2010)

2. Xu, X.-R., Gao, Q.-W., Li, Z.-Y.: Design of wireless sensor networks applied to survey of agriculture environment communication. Transducer and Microsystem Technologies (07) (2009)

3. Zhang, W., Yu, J., Yu, F., Luan, R.: Study on Agricultural Distance Monitoring and Diagnosing Integration Platform Based on XMPP. Chinese Agricultural Science Bulletin (11) (2011)

4. Liu, Z.-S., Wei, F., Chai, Y.-T., Shen, X.-S.: Study on the Construction of the Internet of Things in China. Logistics Technology (07) (2010)

5. Li, G.-G., Li, X.-W., Wen, X.-C.: Influence of Internet of Things Technology on the Development of Automatic Environmental Monitoring System. Environmental Monitoring in China (01) (2011)

6. Liu, H.-J.: Research on Key Technology for Internet of Things. Computer Era (07) (2010)

7. Shen, S.-B., Fan, Q.-L., Zong, P., Mao, Y.-Q., Huang, W.: Study on the Architecture and Associated Technologies for Internet of Things. Journal of Nanjing University of Posts and Telecommunications (Natural Science) (6) (2009)

8. He, K.: The Key Technologies of IOT with Development \& Applications. Radio Frequency Ubiquitous Journal (1) (2010) 